\title{
Rendre visibles l'entreprise, les salariés et les anonymes sur Facebook
}

L'exemple de la communication sur la RSE des firmes du CAC40

\section{Gérald Lachaud et Martine Vila-Raimondi}

\section{(2) OpenEdition \\ Journals}

Édition électronique

URL : http://journals.openedition.org/communicationorganisation/4327

DOI : 10.4000/communicationorganisation.4327

ISSN : 1775-3546

Éditeur

Presses universitaires de Bordeaux

Édition imprimée

Date de publication : 1 décembre 2013

Pagination : 77-86

ISBN : 978-2-86781-878-3

ISSN : $1168-5549$

\section{Référence électronique}

Gérald Lachaud et Martine Vila-Raimondi, «Rendre visibles l'entreprise, les salariés et les anonymes sur Facebook », Communication et organisation [En ligne], 44 | 2013, mis en ligne le 01 décembre 2016, consulté le 19 avril 2019. URL : http://journals.openedition.org/communicationorganisation/4327

DOI : 10.4000/communicationorganisation.4327 


\title{
Rendre visibles l'entreprise, les salariés et les anonymes sur Facebook L'exemple de la communication sur la RSE des firmes du CAC40
}

\author{
Gérald Lachaud' et Mortine Vila-Raimondi ${ }^{2}$
}

L'accomplissement des êtres humains dans une organisation dont la vocation première est surtout financière ne va pas de soi. La communication sur la Responsabilité Sociétale des Entreprises (RSE) proclame pourtant une telle ambition depuis plusieurs années dans des récits qui empruntent à différents univers stylistiques, rhétoriques et sémantiques comme la publicité, la reddition ou la narration. Plusieurs études en communication des organisations ont souligné que ces productions procèdent d'un régime cognitif inédit dans l'économie des savoirs (Loneux, 2010, 56-58), qu'elles visent à instituer la référence au marché comme étalon du lien social (Le Moënne, 2006, 112-113), qu'elles émanent d'un groupe professionnel en cours de constitution et de reconnaissance (de la Broise \& Lamarche, 2010, 67-69) ou, au moins au début, qu'elles s'appuyaient sur des déclarations plutôt que sur des preuves adossées à une certification sociale et environnementale (MartinJuchat, 2007, 44-45). Ces études interrogent pour la plupart des documents écrits tirés de la communication institutionnelle des entreprises (rapports annuels notamment) et n'ont pas toujours intégré les supports numériques, notamment les sites Web dont les cadres successifs d'interprétation des usages et des finalités forgés par l'idéologie managériale n'ont cessé de varier et de diverger (Rouquette, 2009, 256-261). Aussi, à partir des acquis établis par ces études en communication des organisations, nous questionnerons un corpus numérique inédit afin d'étudier quels individus sont mis en visibilité par les entreprises pour leur participation et leur soutien aux actions menées dans le cadre d'une politique de RSE. Nous considérons la visibilité dans

1 Gérald Lachaud est Maître de conférences. Enseignant-chercheur à 1'Université Jean-Moulin Lyon 3, membre d'ELICO Lyon 3. Recherches en cours : la communication numérique des entreprises dans les domaines sociétaux et environnementaux, la responsabilité sociétale des entreprises (RSE).

2 Martine Vila-Raimondi est Maître de conférences. Enseignant-chercheur à l’Université Jean-Moulin Lyon 3, membre d'ELICO Lyon 3. Principaux domaines d'étude : la responsabilité sociétale des entreprises (RSE), la communication numérique d'institutions patrimoniales, les dispositifs pédagogiques dans le cadre de la formation des chargés de communication (bibliothèques, entreprises...). 
son acception classique, comme la qualité pour un individu d'être vu et montré dans un temps et un espace donnés. Par individu, nous entendons toute personne physique identifiable sui generis. Cet individu peut être isolé et anonyme, sympathisant affiché d'un collectif constitué tel qu'une association ou membre d'une organisation telle qu'une entreprise. Nous proposons ainsi d'évaluer la contribution possible de la communication institutionnelle des entreprises au processus de reconnaissance mutuelle, définie comme l'estime sociale accordée aux qualités et capacités concrètes des individus (Honneth, 2000, 147), notamment ceux engagés dans des actions à vocation environnementale, sociale ou sociétale.

\section{Méthodologie}

Trois postulats sous-tendent notre approche. Le premier, d'ordre « technoanthropologique ", argue que les caractéristiques sociales et technologiques de «l'espace de visibilité étendue de l'Internet » (Cardon, 2010, 43) tendent à ériger l'obligation d'immédiateté, d'interactivité et de spontanéité des échanges en principe absolu de toute communication numérique. Le deuxième, d'ordre psychanalytique, considère que "l'imaginaire radical » (Castoriadis, 1975, 532-538) de l'être humain trouve dans les technologies un exutoire à ses désirs, dont celui "d'extimité » défini comme « le désir qui nous incite à montrer certains aspects de notre soi intime pour les faire valider par les autres, afin qu'ils prennent une valeur plus grande à nos propres yeux » (Tisseron, 2011, 121). Le troisième, issu de l'école de la régulation, montre que la RSE est au cœur d'un rapport de force entre travail et capital qui conduit les managers à s'opposer aux exigences exorbitantes des actionnaires au nom de la responsabilité de l'entreprise et à œuvrer en conséquence avec certains acteurs issus de la société civile (Bodet \& Lamarche, 2007).

De ces trois postulats, nous posons les hypothèses suivantes :

- (H1) : la communication numérique externe des entreprises qui se déclarent " responsables " garde les traces visuelles les plus récentes des échanges sur les thèmes de la RSE avec les internautes.

- (H2): cette communication numérique externe est exposée aux difficultés énonciatives rencontrées sur les supports scripturaires. Il s'agit de respecter l'anonymat organisationnel, qui prime sur toute initiative individuelle, tout en assurant la médiatisation des dirigeants qui cherchent à convaincre de la responsabilité de leur entreprise par un discours personnel (de la Broise, 2006, 47-49).

- (H3) : la mise en scène des individus mobilisés par la RSE, qui est opérée par le cadrage et la capture photographiques, puis leur mise en visibilité, qui est réalisée par la communication numérique institutionnelle, relèvent de deux récits. Celui de l'engagement, fondé sur des valeurs universelles qui permettent d'intégrer le projet de l'entreprise dans une mission supérieure au service de l'humanité. Celui de la "maisonnée », basé sur des valeurs internes 
et sur l'identité particulière de la firme, qui contribue à mobiliser le personnel au sein d'une communauté d'appartenance (d'Almeida, 2006, 30-36).

Notre investigation porte sur les quarante sociétés, françaises pour la plupart, qui composent l'indice CAC40 de la Bourse de Paris. Nous avons constitué notre corpus à partir de la consultation du site Web institutionnel de chaque entreprise, afin de retenir celles qui affichaient un «signe passeur " (Davallon \& Jeanneret, 2004, 50) vers une éventuelle page Facebook institutionnelle ou dédiée à la RSE. A la date de figement de notre corpus, le 21 janvier 2013, vingt-six firmes du CAC40 étaient dans ce cas : Accor, Alstom, Axa, BNP Paribas, Bouygues, CAP Gémini, Crédit Agricole, EADS (devenue Airbus), EDF, Essilor, France Télécom (devenue Orange), GDF SUEZ, Gemalto, Lafarge, Legrand, LVMH, PPR (devenue Kering), Publicis, Renault, Safran, Saint-Gobain, Schneider Electric, Société Générale, Technip, Total, VINCI. Cette procédure de figement nous permet de saisir l'instantanéité des fils d'actualité (timeline) et les stratifications successives des posts des internautes opérées par les interventions éditoriales des community managers.

Pour chaque fil d'actualité nous avons étudié la visibilité iconique (photos et avatars) des personnes physiques engagées dans les actions RSE de l'entreprise. Notre perspective est sémio-pragmatique (Odin, 2011, 9-31). Elle prend en compte la situation des acteurs réels (individus) et symboliques (organisations, avatars, etc.) d'après leurs attributs sémiotiques visuels (corporéité et gestualité, proxémique, dispositifs matériels, lieux, etc.). Les 486 images retenues (plus 248 avatars) ont été examinées selon différents critères : fonction organisationnelle supposée des individus (exécution, management, autres parties prenantes), appartenances possibles (ethnie, genre, génération, fonctions et rôles dans la société civile...), nature des situations d'interaction mises en scène (professionnelle, personnelle, sociale), date et période d'intervention et d'inscription dans la timeline. Il faut souligner l'extrême hétérogénéité de ces images, tant du point de vue du contenu, que de la qualité (du cliché d'amateur à la photo extraite d'une banque d'images) ou du format (de l'avatar à la photo très grand format occupant la moitié d'une page-écran). Afin de compléter l'approche iconique, nous avons également, lorsque cela était nécessaire, pris en compte les commentaires textuels affichés sous chaque image du corpus, dès lors qu'ils étaient visibles eux aussi ; c'est-à-dire lisibles dans leur intégralité sans clic supplémentaire de la part de l'internaute.

\section{La visibilité numérique des internautes sous contrôle ?}

Le constat est clair. Si de nombreuses marques recourent à Facebook pour intensifier leur stratégie de relation client, toutes les entreprises du CAC40 ne disposent pas pour l'instant d'une page à vocation institutionnelle. Sans doute redoutent-elles de voir ce support devenir l'exutoire du "désir d'extimité » exhibé à longueur de posts, d'avatars et d'images. D'autant que contrairement aux pages Facebook des marques détenues par les entreprises du CAC40, 
le fil d'actualité des pages institutionnelles de notre corpus ne paraît pas encore être soumis à une veille et à une refonte éditoriale en temps réel. Le Web 2.0, média de l'instantanéité et du refus de la censure, offre donc avant stratification, sédimentation et validation des traces numériques par la communication de l'entreprise une visibilité temporaire, brève et fugace aux anonymes de la toile.

Ainsi, un fil d'actualité d'une entreprise peut conserver les photos de scènes de la vie privée et quotidienne publiées à l'occasion des vœux de fin d'année (Lafarge, le 28/12/12, photos de format moyen occupant un quart de page-écran). Un autre peut contenir le post de personnes en difficulté à la recherche d'un emploi (Total, le 13/13/12). Parfois, des récriminations plus égocentrées apparaissent, comme la photo d'un ticket de caisse publiée par son propriétaire qui s'interroge sur les tarifs d'une station d'essence (Total le 07/12/12). Plus problématiques sans doute pour la réputation de l'entreprise sont les messages qui portent à la connaissance du grand public, nombreuses photos à l'appui, un combat mené par un collectif organisé. Le fil d'actualité devient alors une tribune ouverte. Ainsi, la page Facebook de Lafarge garde les traces visuelles de la lutte de riverains jordaniens d'une installation polluante, capables de manifester devant l'ambassade de France à Aman (18-21/12/12, photos format moyen, un quart de page-écran). Quelques fils conservent pour un temps encore des protestations altruistes portées par un individu isolé, telle cette internaute qui, sous son avatar, interpelle Alstom (" stop to kill our planet ", le 10/01/13) en réponse à un communiqué sur un contrat d'équipement hydroélectrique pour le Vietnam.

Mais passé un délai d'environ quarante-cinq jours maximum (pour Essilor, Lafarge, Legrand et Renault), notre corpus ne garde plus trace d'interventions polémiques d'internautes, repérables et rendues visibles par un avatar ou une photo. Celles qui subsistent encore sont regroupées de façon discrète sous le lien hypertexte standard " commentaires ». Ce qui implique un clic pour les découvrir. Notre hypothèse H1 stipulait que l'affichage d'une " responsabilité » pousserait les entreprises à conserver les traces visuelles d'échanges avec les internautes sur les thèmes de la RSE. Elle semblerait invalidée ici. Nous supposons donc que la plupart des entreprises de notre corpus souhaitent éviter que le fil d'actualité de leur page Facebook ne se transforme en une longue litanie de désapprobations. Considérant sans doute ce dispositif comme un outil de communication d'entreprise à part entière, et non pas comme un média social participatif et ouvert en toute transparence, elles souscriraient ainsi aux vertus du " lissage » (Oger \& Ollivier-Yaniv, $2006,64)$ et des " pratiques de temporalisation » (Carayol, 2005, 85) qui ont cours dans la fabrique du discours institutionnel par la communication des organisations. Parfois ce choix est justifié auprès des internautes, comme le fait VINCI pour le projet Aéroport du Grand Ouest de Nantes (message du 23/10/12). Le risque est alors de ne conserver que les messages et les photos 
à caractère institutionnel émis par le service communication, et de perdre en spontanéité et en popularité.

Toutefois, l'analyse de notre corpus laisserait entrevoir deux autres stratégies possibles.

La première, apparemment adoptée par Renault ou EADS, consisterait à assimiler la page Facebook institutionnelle à une page Facebook dédiée aux fans du groupe industriel dans son ensemble. En effet, le fil d'actualité de ces entreprises comporte en très grande majorité les photos, dans un format conséquent, des nouveaux produits proposés par leurs différentes filiales et entités. La différenciation de cette page institutionnelle avec celles dévolues à chaque marque détenue par l'entreprise pourrait alors être difficile. La seconde stratégie consisterait à dédier une page Facebook à la seule RSE (Axa avec People Protectors, Crédit Agricole avec Agir dans le bon sens), ce qui n'évite pas les interpellations citoyennes et militantes mais dissuade peut-être les posts à caractère privé.

\section{Les parties prenantes invitées sur le fil... d'actualité}

Visibilité comme reconnaissance semblent marquées du sceau de la dialectique : le sens et les enjeux de la relation entre les protagonistes de l'échange symbolique ne sont jamais univoques et définis de manière définitive (Honneth, 2006, 39-100). Les entreprises du CAC40 qui possèdent une page Facebook institutionnelle offrent parfois aux acteurs de la société civile l'opportunité d'être intégrés dans la stratification temporelle du fil d'actualité. Les bénévoles des structures les plus modestes peuvent alors bénéficier d'une visibilité inattendue. Les membres d'une association britannique érigeant un monument commémoratif aux pilotes de la RAF morts dans un crash en 1943 sont ainsi à l'affiche du fil d'actualité de Lafarge, généreuse donatrice d'un bloc de granit (23/11/11, photo de format moyen).

Parmi les entreprises du CAC40 qui choisissent de créer une fondation pour orienter les actions de mécénat sociétal, deux options semblent possibles. D'un côté, Bouygues réserve apparemment la priorité, voire l'exclusivité, de la reconnaissance et de la visibilité du fil d'actualité aux individus aidés par cette seule fondation. D'un autre côté, Crédit Agricole ouvre son fil d'actualité aux représentants d'associations soutenues par des caisses et des succursales locales. Cette divergence constatée s'explique sans doute par des choix de stratégie de communication, mais aussi par une centralisation structurelle probablement plus poussée dans un groupe industriel fondé par un self-made man du BTP que dans un réseau bancaire fédéral issu du mouvement mutualiste.

Est-ce pour échapper à la critique d'égoïsme et d'indifférence, mais aussi pour respecter les axiomes de commune humanité et de commune dignité entre « grands » et " petits» qui sous-tendent la justification de l'action dans les différents mondes sociaux, que certaines entreprises partagent ainsi leur notoriété (Boltanski \& Thévenot, 1991, 96-102) ? 
À l'inverse, d'autres préféreraient bénéficier de l'aura des institutions internationales, comme en témoigne le drapeau onusien affiché en très grand format (la moitié d'une page-écran) dans le fil d'actualité de LVMH à l'année 2003 pour rappeler que cette entreprise est signataire du Pacte Mondial. De même, quelques personnalités médiatiques plus ou moins célèbres, sont visibles sur les fils d'actualité. Elles sont issues des arts ou du sport, plus rarement de la politique. Il est vrai aussi, dans ce dernier cas, que les implicites idéologiques de la RSE tendent à ignorer pour le moins les États et à privilégier d'autres parties prenantes (Capron \& Quairel-Lanoizelée, 2004, 31-40).

\section{La présence fugace des instances dirigeantes}

Notre hypothèse $\mathrm{H} 2$ postulait un affichage certain des dirigeants d'entreprise pour convaincre de la responsabilité de leur entreprise. Tel n'est pas le cas dans notre corpus. Même les entreprises les plus empreintes de la réussite individuelle de leur fondateur (Bouygues, PPR, LVMH) ne mettent pas en avant cette figure tutélaire. Les raisons tiennent peut-être autant à la volonté des personnes elles-mêmes, qu'aux conseils des spécialistes de l'image ou aux préconisations des responsables de la communication soucieux d'établir la distinction entre ce qui relève de la sphère privée et de la vie institutionnelle de l'entreprise. Seule exception à ce constat empirique : le fil d'actualité de Publicis dont le PDG, Maurice Lévy, paraît à dix-huit reprises, soit dans près du tiers des visuels recensés, mais à de très rares exceptions au titre de la responsabilité sociétale de son entreprise. En comparaison, le très emblématique Bernard Arnault n'apparaît jamais en photo sur le fil d'actualité de la page LVMH et il n'est cité que quatre fois. De la même manière, le PDG de Schneider Electric, Jean-Pascal Tricoire, n'est montré qu'une seule fois, mais à l'occasion d'un entretien préalable à la signature d'un accord de son entreprise pour la fabrication de lampes basse consommation, en compagnie du prestigieux Prix Nobel de la Paix Muhammad Yunus (20/06/13 photo format moyen).

Même parcimonieuse, la mise en visibilité institutionnelle des dirigeants au titre de la responsabilité sociétale de l'entreprise qu'il dirige privilégierait donc plutôt les contextes officiels et institutionnels. Mais elle peut aussi se satisfaire pour la bonne cause d'une situation beaucoup plus modeste et informelle. Le PDG d'Essilor apparaît ainsi deux fois sur le fil d'actualité. La première le montre en tant que président de la Fondation Essilor recevant une distinction accordée par le Special Olympics Fundation à Washington (les 11/10/12 et 19/09/12, photos format moyen). La seconde le met en scène photographié aux côtés de deux autres personnes (des collaborateurs ?), dans un lieu public de type galerie marchande, participant en T-shirt à un happening en faveur de la Journée mondiale de la vue.

Mais les chefs d'entreprise ne sont pas les seuls à avoir droit aux honneurs de la communication numérique de leur entreprise. 


\section{L'investissement des salariés dans la RSE récompensé et célébré}

Trois types de dispositifs de reconnaissance de l'engagement des collaborateurs dans les actions RSE de l'entreprise sont observables suite à notre étude.

Le premier rassemble les rituels de la vie de l'organisation (conventions, cérémonies, galas...). Le fil d'actualité le plus représentatif à cet égard est celui du groupe Bouygues qui expose chaque année les visages souriants de la " diversité "soutenus par sa fondation, soit à la tribune, micro en main, soit dans les coulisses de la cérémonie de gala, en compagnie de leur parrain dans l'entreprise (27/10/11 photo très grand format). De la même manière, le fil d'actualité de Saint-Gobain réitère de manière régulière à six reprises en quinze mois les posts accompagnés de photos de format réduit qui rendent compte d'attributions de prix honorifiques pour ses salariés dans les domaines de l'environnement, des ressources humaines ou du social.

Le deuxième type de dispositif regroupe toutes les interventions directes ou indirectes des salariés en faveur d'une cause (actions sur le " terrain ", opérations de sensibilisation, collectes de fonds...). Lafarge s'illustre ici tout particulièrement. En effet, à l'opposé de Bouygues, ses salariés sont photographiés lors de challenges sportifs destinés à récolter des fonds pour la recherche médicale (08/10/10 photo format moyen), lors de journées de nettoyage de sites naturels (08/11/12 photo format moyen), ou encore lors d'un chantier de construction solidaire en Roumanie (03/11/10 photo format moyen).

Le dernier type de dispositif, constaté avec plus de rareté, réunit les situations où les salariés sont montrés dans l'exercice d'une activité de production qualifiée de responsable par les commentaires de la communication institutionnelle de l'entreprise. Renault et Alstom sont ici emblématiques dans leur choix opposé. Leur fil d'actualité est avant tout illustré par les photos de leurs produits (voitures ou trains) présentés lors d'un lancement officiel ou mis en scène en situation d'usage. Mais la communication numérique institutionnelle d'Alstom diverge de celle de Renault. En effet, les individus sont très peu présents dans les photos postées et plus rarement encore les salariés. À l'inverse, Renault n'hésite pas à reconnaître la contribution environnementale de ses collaborateurs, dès lors qu'ils posent fièrement assemblés devant la chaîne de montage du $5000^{\mathrm{e}}$ véhicule électrique Kangoo (17/04/12 photo pleine page écran). Notre hypothèse $\mathrm{H} 3$ stipulait l'entremêlement de deux récits. Celui de l'engagement, qui met le projet de l'entreprise au service de l'humanité par le recours à des valeurs universelles. Celui de la "maisonnée ", qui mobilise le personnel dans une communauté fondée sur une identité particulière. La double reconnaissance $a u$ travail et $d u$ travail proposée par la communication numérique externe de Renault constitue l'un des rares indices visuels de notre corpus confirmant cette hypothèse. 
Certains salariés qui s'investissent dans des actions RSE de leur entreprise se voient donc octroyer par les services de communication une visibilité et une reconnaissance durables, puisqu'inscrites dans la temporalité longue des fils d'actualité. Ainsi, à la figure d'un quidam, certes visible pour un bref moment, isolé et réduit au statut d'avatar ou de dépositaire de photos d'amateur, s'opposerait l'image, mise en scène et travaillée par la communication de l'entreprise, de personnes choisies pour leurs actions en faveur de la RSE, insérées dans un groupe et reconnues pour leur capacité à maîtriser toutes les ressources du « déploiement de soi » (Jauréguiberry, 2011, 138-141).

La création d'une page Facebook institutionnelle peut constituer pour une entreprise l'opportunité d'augmenter la visibilité de ses parties prenantes et la possibilité de favoriser le partage de ses valeurs. Cependant, l'examen de notre corpus portant sur les vingt-six firmes du CAC40 qui disposaient d'une telle page début 2013 interroge cette opportunité et cette possibilité. Questionnement que le suivi dynamique en temps réel des fils d'actualité d'entreprises ainsi que des entretiens avec les acteurs eux-mêmes pourraient contribuer à résoudre.

Cependant, le développement encore lent des pages Facebook institutionnelles, les apparentes divergences dans les choix éditoriaux des fils d'actualité et l'absence d'homogénéité dans la mise en visibilité des salariés au titre de leur engagement dans la RSE témoigneraient, selon nous, d'un doute des décideurs et des communicants. Il pourrait aussi attester d'une réflexivité critique d'une partie de la sphère managériale soucieuse de prendre ses distances avec des pratiques de "reconnaissance stratégique " (Heller, 2009, 102-106), consistant à prêter aux individus des qualités pour mieux obtenir d'eux une performance.

Mais de la même manière, la relative discrétion des posts les plus récriminatoires, avant la refonte éditoriale des community managers, attesterait d'une distance critique des internautes désireux de publiciser leur combat ou de contester l'idée même d'entreprise responsable, sans devoir participer pour autant à La fabrique des imposteurs (Gori, 2013) que seraient, selon eux, certains réseaux sociaux numériques, comme par exemple le fil d'actualité d'une page Facebook institutionnelle.

La volonté des anonymes d'être agrégés au flux temporel de l'organisation, symbolisé par le fil d'actualité, comme l'intention des services de communication d'associer à ce même flux les salariés investis dans la RSE, serait peut-être l'indice d'un malaise de la temporalité plus profond, défini comme "une difficulté inédite de nous envisager dans la durée ou plus exactement de nous inscrire - en tant qu'individus - dans la trame d'un temps collectif » (Zawadzki, 2011, 12-13). 


\section{BIBLIOGRAPHIE}

ALMEIDA N. d', La perspective narratologique en organisation, in de la BROISE P., LAMARCHE Th. Coord., Responsabilité sociale : vers une nouvelle communication des entreprises? Villeneuve d'Ascq : Septentrion, 2006, p. 27-38.

BROISE P. de la, Entre reddition et légitimation, le rapport annuel d'entreprise, in de la BROISE P., LAMARCHE Th. Coord., Responsabilité sociale : vers une nouvelle communication des entreprises? Villeneuve d'Ascq : Septentrion, 2006, p. 39-51.

BODET C., LAMARCHE Th., La responsabilité sociale des entreprises comme innovation institutionnelle : une lecture régulationniste, in Revue de la régulation, 2007, $\mathrm{n}^{\circ} 1$, [en ligne] http://regulation.revues.org/1283

BOLTANSKI L., THÉVENOT L., De la justification. Les économies de la grandeur. Paris : Gallimard, 1991, 483 p.

CAPRON M., QUAIREL-LANOIZELÉE F., Mythes et réalités de l'entreprise responsable. Acteurs, enjeux, stratégies. Paris : La Découverte, 2004, 252 p.

CARDON D., La démocratie Internet. Promesses et limites. Paris : Le Seuil, 2010, 102 p.

CARAYOLV.,Principe de contrôle, communication et temporalités organisationnelles, in Études de communication, 2005, n ${ }^{\circ} 28$, p. 77-89.

CASTORIADIS C., L'institution imaginaire de la société. Paris : Le Seuil, 1975, 542 p. DAVALLON J., JEANNERET Y., La fausse évidence du lien hypertexte, in Communication et langages, 2004, $\mathrm{n}^{\circ} 140,43-54$.

GORI R., La fabrique des imposteurs. Paris : Les liens qui libèrent, 2013, 313 p.

HONNETH A., La lutte pour la reconnaissance. Paris, Le Cerf, 2000, 232 p.

HONNETH A., La société du mépris. Vers une nouvelle théorie critique. Paris, La Découverte, 2006, 349 p.

HELLER Th., Reconnaissance et gouvernement des salariés. Au-delà du mépris, in Questions de communication, 2009, n 15, p. 93-107.

JAURÉGUIBERRY F., L'exposition de soi sur Internet : un souci d'être au-delà du paraitre, in AUBERT N., HAROCHE C. Coord., Les tyrannies de la visibilité. Être visible pour exister? Toulouse : Eres, 2011, p. 131-144.

LE MOËNNE Ch., Les communications d'entreprise entre médias, réseaux et recompositions organisationnelles, in BOUZON A. Coord. La communication organisationnelle en débat : champs, concepts, perspectives. Paris : L'Harmattan, 2006, p. 103121.

LONEUX C., Enjeux de gouvernance à l'épreuve des discours de la RSE, in Communication \& Organisation, 2010, n 37, p. 53-63.

MARTIN-JUCHAT F., Communication des entreprises sur la responsabilité sociale : constat du décalage français, in Les enjeux de l'information et de la communication, 2007, n 1 p. 35-49.

ODIN R., Les espaces de communication. Introduction à la sémio-pragmatique. Grenoble : PUG, 2011, $159 \mathrm{p}$.

OGER C., OLLIVIER-YANIV C., Conjurer le désordre discursif. Les procédés de « lissage » dans la fabrication du discours institutionnel, in Mots, 2006, n 81, p. 63-77. 
ROUQUETTE S., L'analyse des sites internet. Une radiographique du cyberespace. Bruxelles/Paris : De Boeck/INA, 2009, 335 p.

TISSERON S., Les nouveaux réseaux sociaux : visibilité et invisibilité sur le net, in AUBERT N., HAROCHE C. Coord., Les tyrannies de la visibilité. Être visible pour exister? Toulouse : Eres, 2011, p. 119-129.

ZAWADZKI P., Malaise dans la temporalité. Dimensions d'une transformation anthropologique silencieuse, in ZAWADZKI P. Coord. Malaise dans la temporalité. Paris : Publications de la Sorbonne, 2002, p. 9-66.

Résumé : Cet article traite de la communication numérique des entreprises dans le domaine de la RSE. Il s'appuie sur l'étude du fil d'actualité du compte institutionnel Facebook des firmes du CAC40. Il souligne que les pratiques éditoriales des responsables de la communication et des webmasters ouvrent, pour un temps limité, ce support aux anonymes de l'internet. Il étudie les différentes formes de présence et de visibilité des acteurs de la société civile (associations). Il interroge ensuite la relative discrétion de la figure dirigeante. Il montre enfin la place accordée à la reconnaissance des salariés engagés et investis dans des actions de RSE.

Mots-clés : communication numérique, visibilité, entreprises, CAC 40, reconnaissance, engagement, organisation.

\begin{abstract}
This article deals with the digital communication of firms as far as Corporate Social Responsibility is concerned. It relies on the study of the feeds of the institutional accounts of the firms of the CAC 40. It highlights how the publishing practises of the webmasters and managers of the communication open up, for a limited duration, this support to anonymous contributors on the Internet. It studies the different aspects of the presence and visibility of the actors of civil society (associations). It then questions the relative discretion of the management team. It finally shows how recognition is awarded to the employees who commit themselves and are engaged in the process of Corporate Social Responsibility.
\end{abstract}

Abstract: digital communication, visibility, firms, $C A C$ 40, recognition, commitment, organisation. 